\title{
Optical properties of graphene antidot lattices
}

Pedersen, Thomas Garm; Flindt, Christian; Pedersen, Jesper Goor; Jauho, Antti-Pekka; Mortensen, Asger; Pedersen, Kjeld

Published in:

Physical Review B Condensed Matter

Link to article, DOI:

10.1103/PhysRevB.77.245431

Publication date:

2008

Document Version

Publisher's PDF, also known as Version of record

Link back to DTU Orbit

Citation (APA):

Pedersen, T. G., Flindt, C., Pedersen, J. G., Jauho, A-P., Mortensen, A., \& Pedersen, K. (2008). Optical properties of graphene antidot lattices. Physical Review B Condensed Matter, 77(24), 245431.

https://doi.org/10.1103/PhysRevB.77.245431

\section{General rights}

Copyright and moral rights for the publications made accessible in the public portal are retained by the authors and/or other copyright owners and it is a condition of accessing publications that users recognise and abide by the legal requirements associated with these rights.

- Users may download and print one copy of any publication from the public portal for the purpose of private study or research.

- You may not further distribute the material or use it for any profit-making activity or commercial gain

- You may freely distribute the URL identifying the publication in the public portal

If you believe that this document breaches copyright please contact us providing details, and we will remove access to the work immediately and investigate your claim 


\title{
Optical properties of graphene antidot lattices
}

\author{
Thomas G. Pedersen, ${ }^{1}$ Christian Flindt, ${ }^{2}$ Jesper Pedersen, ${ }^{2}$ Antti-Pekka Jauho, ${ }^{2,3}$ Niels Asger Mortensen, ${ }^{2}$ and Kjeld Pedersen ${ }^{1}$ \\ ${ }^{1}$ Department of Physics and Nanotechnology, Aalborg University, DK-9220 Aalborg Ø, Denmark \\ ${ }^{2}$ Department of Micro and Nanotechnology, NanoDTU, Technical University of Denmark, DK-2800 Kongens Lyngby, Denmark \\ ${ }^{3}$ Laboratory of Physics, Helsinki University of Technology, P.O. Box 1100, 02015 HUT, Finland \\ (Received 14 February 2008; revised manuscript received 30 April 2008; published 23 June 2008)
}

\begin{abstract}
Undoped graphene is semimetallic and thus not suitable for many electronic and optoelectronic applications requiring gapped semiconductor materials. However, a periodic array of holes (antidot lattice) renders graphene semiconducting with a controllable band gap. Using atomistic modeling, we demonstrate that this artificial nanomaterial is a dipole-allowed direct-gap semiconductor with a very pronounced optical-absorption edge. Hence, optical infrared spectroscopy should be an ideal probe of the electronic structure. To address realistic experimental situations, we include effects due to disorder and the presence of a substrate in the analysis.
\end{abstract}

DOI: 10.1103/PhysRevB.77.245431

PACS number(s): 78.67.-n, 73.22.-f, 73.61.Wp

\section{INTRODUCTION}

Graphene has emerged as a promising material for nanoscale electronic devices. Most importantly, graphene combines a high mobility $\left[\sim 15000 \mathrm{~cm}^{2} / \mathrm{V} \mathrm{s}\right.$ (Refs. 1 and 2)] with the possibility of patterning using e-beam lithography. ${ }^{2-4}$ In addition, the very long spin-coherence time is important for potential spintronics applications. ${ }^{5,6}$ Patterning of graphene into Hall bars, ${ }^{1,7}$ quantum dots, ${ }^{2,3}$ nanoribbons, ${ }^{4}$ and circular Aharonov-Bohm interferometers ${ }^{8}$ has been demonstrated. Recently, we have proposed adding graphene antidot lattices ${ }^{9}$ to this list. Our proposed antidot structure consists of a hexagonal array of circular holes perforating the graphene sheet. Such a periodic perturbation turns the semimetallic sheet into a semiconductor with a controllable band gap. Furthermore, "defects" in the lattice formed by leaving one or several unit cells intact support localized states that could lead to realization of a graphene spin qubit architecture. ${ }^{9}$ However, the fully periodic antidot lattice is highly interesting in itself. For instance, transport under magnetic fields could lead to Hofstadter butterfly features. ${ }^{10}$ Also, the tunable band gap could be used to design quantum wells and channels for electronic devices. It is even conceivable that tunable absorption and emission of light could lead to novel graphene optoelectronic devices.

In this work, we present a theoretical study of the optical properties of graphene antidot lattices. Expanding on our previous work, ${ }^{9}$ we demonstrate how optical spectroscopy will be useful in characterizing the electronic structure of antidot lattices. In particular, we predict a highly visible absorption edge corresponding to the band gap. Hence, optical (infrared) absorption spectroscopy is a promising candidate for characterization. We compute the optical properties using a tight-binding formalism. ${ }^{11}$ To accelerate convergence with respect to $k$-point sampling, an improved triangle integration method including $k$-dependent matrix elements has been developed. We present a systematic study of the absorption signature in two different families of lattices as the size of the perforation increases. In practice, variation in hole position and size/shape will lead to inhomogeneously broadened spectra. We study the influence of broadening on the optical spectra to gauge the effect on the measurable response. Also, samples placed on substrates are considered. We find that even in the presence of broadening, both absorption and reflection contrast spectra display clearly detectable band-gap features. Finally, the dependence of the low-frequency refractive index on energy gap is analyzed for a large compilation of antidot structures.

\section{THEORY AND METHODS}

The optical properties of an extremely thin layer, such as monolayer graphene, can be characterized in two distinct ways. Physically, it is appropriate to view the layer as a charge sheet with complex sheet conductivity $\widetilde{\sigma}(\omega)$. Alternatively, the sheet may be viewed as a homogeneous layer with a small but finite thickness $d_{g}$, taken as the graphite interlayer lattice constant $\sim 3.35 \AA$, and characterized by a dielectric constant $\varepsilon(\omega)$. As long as the layer thickness is much less than the wavelength, the two approaches lead to virtually identical results provided the response functions are related via $\widetilde{\sigma}(\omega)=-i d_{g} \varepsilon_{0} \omega[\varepsilon(\omega)-1]$. The antidot lattice is a periodic structure and as such all properties are calculated as appropriate integrals over a two-dimensional Brillouin zone. We apply the following approach in all computations: First, the limit of vanishing broadening is considered. This allows us to calculate the real part of the conductivity using a highly accurate triangle integration method including $k$-dependent matrix elements. The details of this method are given in Appendix. Second, the imaginary part of the conductivity is obtained via a Kramers-Kronig transform. Finally, broadening is reintroduced by convoluting with a Gaussian line broadening function. We consider only fully periodic structures and ignore exciton effects in the present work. Localized excitons produce additional discrete absorption resonances below the band gap and the continuous spectrum above the gap is modified by continuum excitons. By ignoring electron-hole interaction, we disregard discrete resonances and approximate the continuous spectrum by the single-electron response. The single-electron response is sufficiently complex and computationally demanding that we choose to postpone exciton effects to future work, however. 
Following Ref. 12, the real part of the conductivity $\sigma(\omega)$ $=\operatorname{Re} \widetilde{\sigma}(\omega)$ at low temperature is

$$
\sigma(\omega)=\frac{e^{2}}{2 \pi m^{2} \omega} \sum_{v, c} \int\left|P_{v c}\right|^{2} \delta\left[E_{c v}(\vec{k})-\hbar \omega\right] d^{2} k,
$$

where $P_{v c}$ is the in-plane momentum matrix element and $E_{c v} \equiv E_{c}-E_{v}$ is the transition energy between valence band $v$ and conduction band $c$. This expression applies to regular graphene as well as graphene antidot lattices provided the integral is taken over appropriate Brillouin zones. Energies and matrix elements are computed from tight-binding eigenstates. We use a simple orthogonal $\pi$-electron model with a nearest-neighbor transfer integral of $\gamma=3.033 \mathrm{eV} .{ }^{13}$ This model is known to agree with the first-principles band structure in the low-energy range. Corrections for edge effects can be incorporated into the transfer integral but lead only to a slight opening of the antidot band gap. ${ }^{9}$ The momentum operator is given solely by the $k$-space gradient of the tightbinding Hamiltonian $\vec{P}=(m / \hbar) \nabla_{k} H$ since intra-atomic terms are absent in $\pi$-electron models. ${ }^{11}$

As a reference, we first consider a regular graphene sheet without an antidot lattice. In this case, the analytic results of Ref. 12 (correcting typographical errors) yield a conductivity

$$
\begin{aligned}
\sigma(\omega)= & \frac{e^{2}}{\sqrt{24} \pi \hbar \Omega^{3 / 2}} \operatorname{Re}\left[\frac{144-12 \Omega+\Omega^{3}}{24} K\left(\frac{(6-\Omega)(2+\Omega)^{3}}{128 \Omega}\right)\right. \\
& \left.-12 E\left(\frac{(6-\Omega)(2+\Omega)^{3}}{128 \Omega}\right)\right]
\end{aligned}
$$

where $\Omega=\hbar \omega / \gamma$ and $K$ and $E$ are elliptic integrals. It can be shown that taking the zero-frequency limit of the above expression leads to a minimum graphene conductivity of $\sigma$ $=\sigma_{0} \equiv e^{2} / 4 \hbar$ in agreement with several other calculations. ${ }^{14,15}$ Retaining the first nonvanishing correction one finds $\sigma \approx \sigma_{0}\left(1+\Omega^{2} / 9\right)$ at low frequencies. The lowfrequency response is modified if the chemical potential is shifted away from the Dirac point via doping ${ }^{16}$ but in the present work only intrinsic graphene is considered. To illustrate the accuracy of the improved triangle method we compare in Fig. 1 the exact result given by Eq. (2) to numerical integration based on (a) the triangle method with $153 k$ points and (b) simple rectangular discretization of Eq. (1) using $5050 k$ points and a broadening of $20 \mathrm{meV}$. Numerical integration is taken over the irreducible Brillouin zone using a symmetrized matrix element $\left|P_{v c}\right|^{2}=\left(\left|P_{v c}^{x}\right|^{2}+\left|P_{v c}^{y}\right|^{2}\right) / 2$. Even with only a fraction of the $k$ points, the triangle integration is clearly superior to simple discretization. Moreover, the agreement with the exact curve is excellent.

\section{RESULTS}

We now turn to antidot lattices in which an energy gap opens around the Fermi level. As demonstrated in Ref. 9, perforation of a graphene sheet by a regular hexagonal array of circular holes yields a gapped band structure that can be controlled to a large extent by varying the radius and distance between holes. In addition, hole shape may play an important role in determining the properties. For instance,

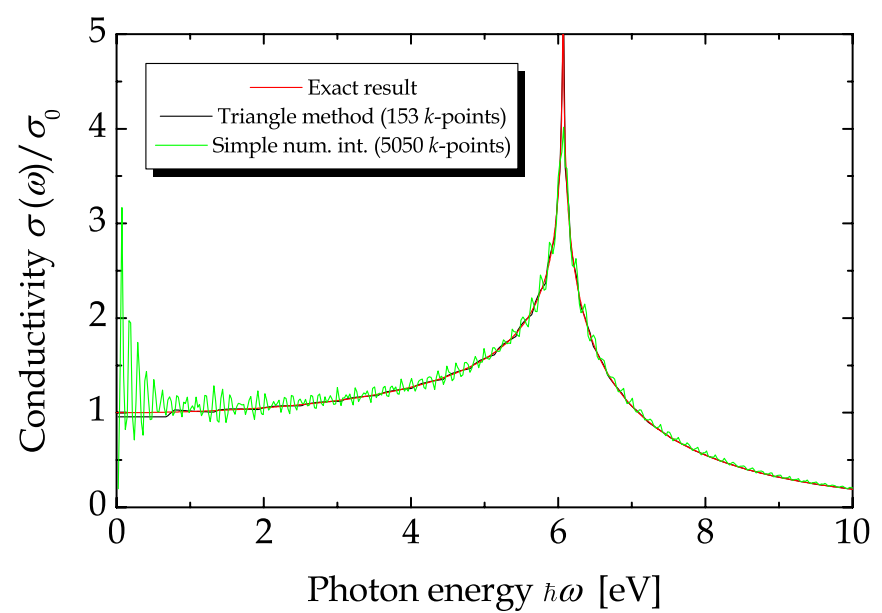

FIG. 1. (Color online) Comparison of numerical triangle integration with the exact conductivity and simple discretization. Note the different $k$-point sampling for the two numerical schemes.

replacing the circular perforation with a triangular one having zigzag edges produces a dispersionless "metallic" band at the Fermi level. The different band structures are illustrated in Fig. 2. Here, the circular structure is a $\{10,3\}$ antidot lattice in the $\{L, R\}$ notation suggested in Ref. 9: $L$ is the side length and $R$ the radius of the perforation, both in units of the graphene lattice constant. The unit cell of the triangular structure is similar in size to the circular case and the area of the triangular perforation is roughly equal to that of the circular hole. The bandwidth of the dispersionless band is identically zero because the simple nearest-neighbor model allows for eigenstates in which the node-structure completely decouples all occupied $\pi$ orbitals in the zigzag case. If interactions beyond nearest neighbors are included, a small but finite bandwidth is observed. Antidot lattices with such triangular perforations would lead to additional interesting features in the optical response such as controllable transparency windows. Presumably, their fabrication using, e.g., e-beam lithography will be rather demanding, however, and in the remaining part of the paper we focus on circular perforations.

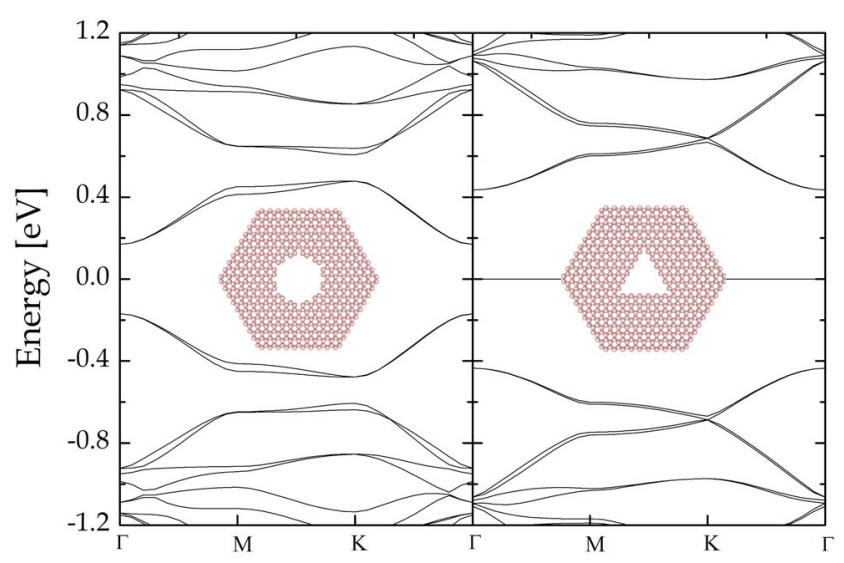

FIG. 2. (Color online) Band structures of a $\{10,3\}$ antidot lattice and similar structure having a triangular hole with zigzag edges. Note the dispersionless band at $0 \mathrm{eV}$ in the triangular case. 


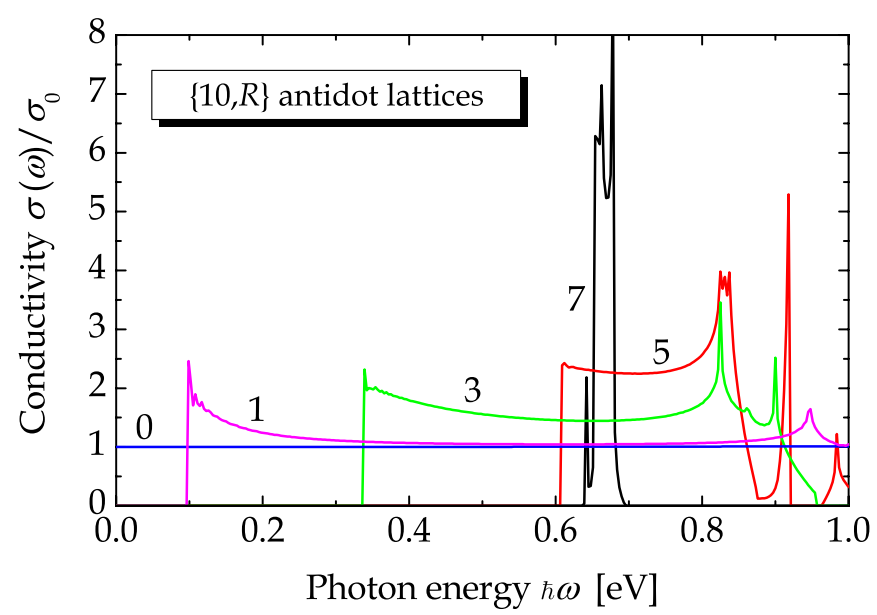

FIG. 3. (Color online) Conductivity spectra for several $\{10, R\}$ antidot lattices with $R$ indicated next to each spectrum. The conductivity is normalized to the dc value $\sigma_{0}=e^{2} / 4 \hbar$.

For numerical integration, the irreducible Brillouin zone is partitioned into 4098 triangles, which is equivalent to 2145 unique $k$ points. In usual two-dimensional direct band-gap semiconductors with parabolic energy dispersion, the absorption edge is a clearly discernable step profile. ${ }^{17}$ Our numerical results show that a similar behavior is found in graphene antidot lattices. As an illustration, in Figs. 3 and 4 the conductivity spectra are shown for $\{10, R\}$ and $\{12, R\}$ lattices. The steplike absorption edge coincides with the band gap and demonstrates that antidot lattices are two-dimensional dipole-allowed direct-gap semiconductors. This will be important for possible optoelectronic applications including light emission and detection. Also, experimental detection of band gaps using infrared spectroscopy should be feasible with such a clear signature.

To fully characterize the optical properties we need to determine both real and imaginary parts of the frequencydependent conductivity. Also, inhomogeneous broadening must be considered as practical e-beam patterning will lead to variations in hole size, shape, and position. The imaginary part of $\widetilde{\sigma}(\omega)$ is readily obtained from a Kramers-Kronig

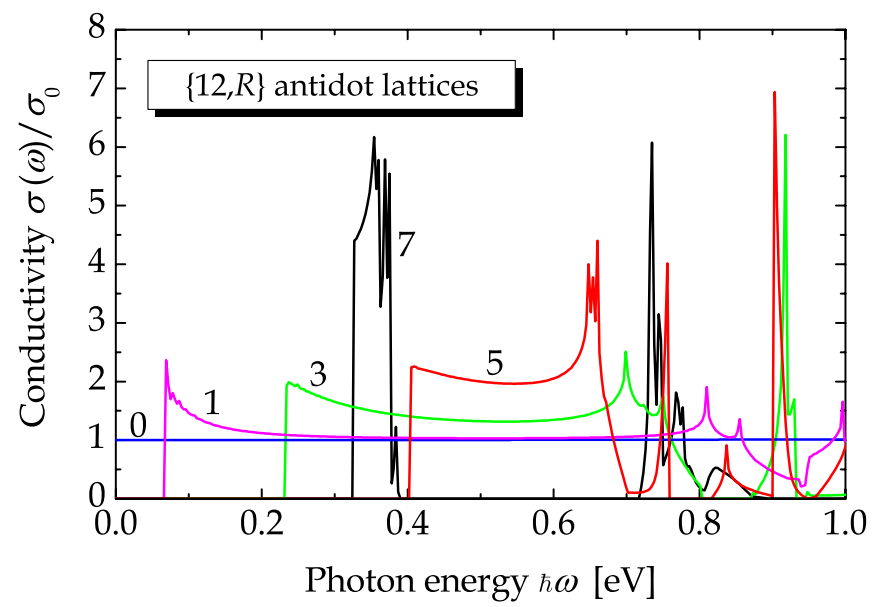

FIG. 4. (Color online) Same as Fig. 3 but for the $\{12, R\}$ family of lattices.

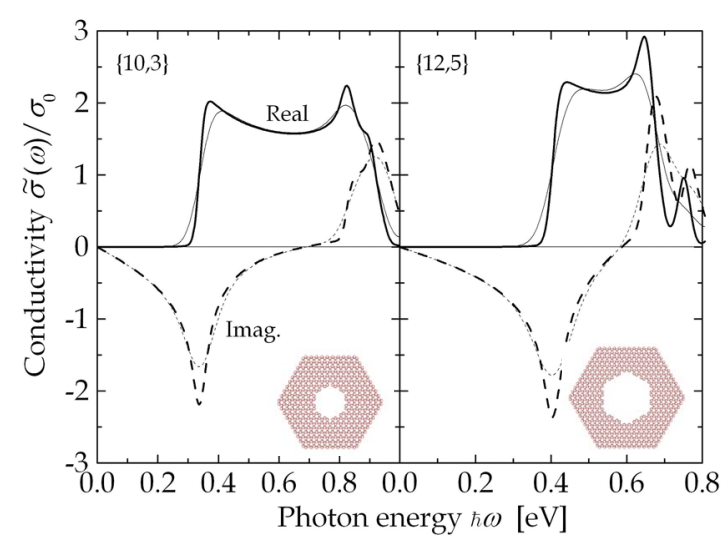

FIG. 5. (Color online) Complex conductivity spectra including broadening. Curves are real parts (solid lines) and imaginary parts (dashed lines) for $\hbar \Gamma=20 \mathrm{meV}$ (thick lines) and $\hbar \Gamma=50 \mathrm{meV}$ (thin lines).

transform of the real part. Care should be taken, however, that the real part is calculated up to sufficiently large frequencies. Subsequently, broadening can be included by convoluting with a Gaussian line-shape function $\exp [-(\omega$ $\left.\left.-\omega^{\prime}\right)^{2} / \Gamma^{2}\right] /(\Gamma \sqrt{\pi})$. The broadening $\Gamma$ reflects the degree of disorder and we estimate that high quality samples should have $\hbar \Gamma<100 \mathrm{meV}$. In Fig. 5, we show the effect on the complex conductivity of broadening by $\hbar \Gamma=20$ and $50 \mathrm{meV}$. Increased broadening tends to blur finer features in the spectra but at this level of disorder the absorption edge is still clearly visible. We emphasize that it is the steplike absorption edge of the two-dimensional semiconductor that makes band-edge detection feasible for samples with relatively low disorder.

In practical experiments, graphene samples are usually positioned on a suitable substrate for investigations. Hence, it is of importance to discuss the role of substrates on the optical signatures of antidot lattices. For transmission measurements any transparent substrate can be used and the recorded spectrum will essentially provide the real part of the conductivity directly. Alternatively, a reflection geometry can be used. Usually, an oxidized silicon wafer is applied for this purpose. In fact, monolayer graphene is usually identified in mechanically peeled graphite flakes by observing flakes of varying thickness on oxidized $\mathrm{Si}$ wafers in an optical microscope. ${ }^{1,2}$ Using white light illumination and an oxide thickness of $300 \mathrm{~nm}$, it turns out that even monolayer graphene is clearly visible in the microscope. The contrast, which is around $15 \%$, is a result of a fortuitous choice of oxide thickness and the large conductivity of graphene. ${ }^{18-20}$ The geometry of such a sample is illustrated in Fig. 6. We denote the frequency-dependent refractive indices of $\mathrm{SiO}_{2}$ and Si by $n_{1}$ and $n_{2}$, respectively, and the oxide thickness by $d$. Introducing a dimensionless graphene conductivity $\bar{\sigma}$ $\equiv \widetilde{\sigma} / \varepsilon_{0} c$ the reflectance at normal incidence is given by

$$
R=\left|\frac{e^{2 i d n_{1} \omega / c}\left(1+n_{1}-\bar{\sigma}\right)\left(n_{1}-n_{2}\right)+\left(1-n_{1}-\bar{\sigma}\right)\left(n_{1}+n_{2}\right)}{e^{2 i d n_{1} \omega / c}\left(1-n_{1}+\bar{\sigma}\right)\left(n_{1}-n_{2}\right)+\left(1+n_{1}+\bar{\sigma}\right)\left(n_{1}+n_{2}\right)}\right|^{2} .
$$

A general expression valid at arbitrary angle of incidence is given in Ref. 20. The reflectance contrast is defined as $\left(R_{0}\right.$ 


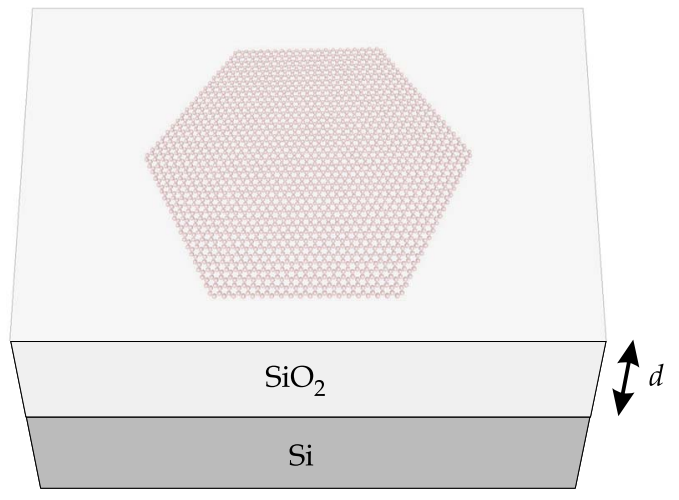

FIG. 6. (Color online) Graphene sample positioned on an oxidized $\mathrm{Si}$ wafer.

$-R) / R_{0}$, where $R_{0}$ is calculated as above but taking $\bar{\sigma}=0$. We take experimental refractive indices of $\mathrm{SiO}_{2}$ and $\mathrm{Si}$ from Refs. 21 and 22, respectively.

In Fig. 7, we have displayed the reflectance contrast of $\{10,3\}$ and $\{12,5\}$ antidot lattices positioned on $300 \mathrm{~nm}$ oxide $\mathrm{Si}$ wafers. In the computation, the complex conductivity spectra shown in Fig. 4 for the case $\hbar \Gamma=20 \mathrm{meV}$ have been applied. It is apparent that a large contrast exceeding $20 \%$ is predicted for this situation. In the inset, the contrast for regular graphene in the visible is illustrated for comparison. The magnitude and location of the resonance just below $600 \mathrm{~nm}$ are in good agreement with experiments and other calculations. ${ }^{18}$ For the $\{10,3\}$ and $\{12,5\}$ structures the band gaps are around 0.34 and $0.41 \mathrm{eV}$, respectively (cf. Figs. 3 and 4). This corresponds to resonance wavelengths of 3650 and $3025 \mathrm{~nm}$. These resonances are clearly observed in the contrast plots in Fig. 7. Hence, reflectance contrast measurements could be a viable method of determining band edges. Recently, optical spectroscopy on gated graphene in precisely this wavelength range has been reported, ${ }^{23}$ which further supports the feasibility of our proposal.

Several optical and electro-optic applications of graphene antidot lattices can be envisioned. For instance, light emitting devices tailored to specific wavelengths could be fabricated. It might also be possible to incorporate antidot lattices

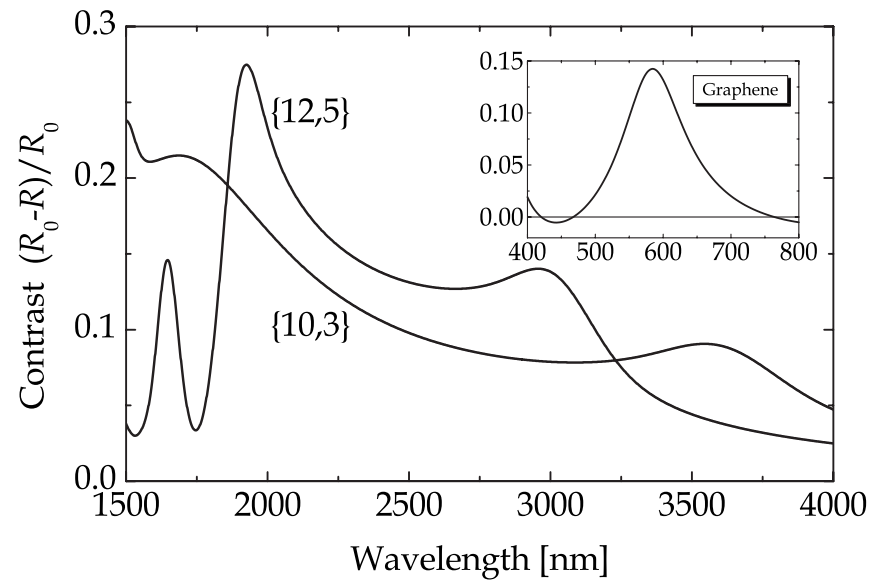

FIG. 7. Infrared reflectance contrast for $\{10,3\}$ and $\{12,5\}$ lattices. Inset: contrast for regular graphene in the visible.

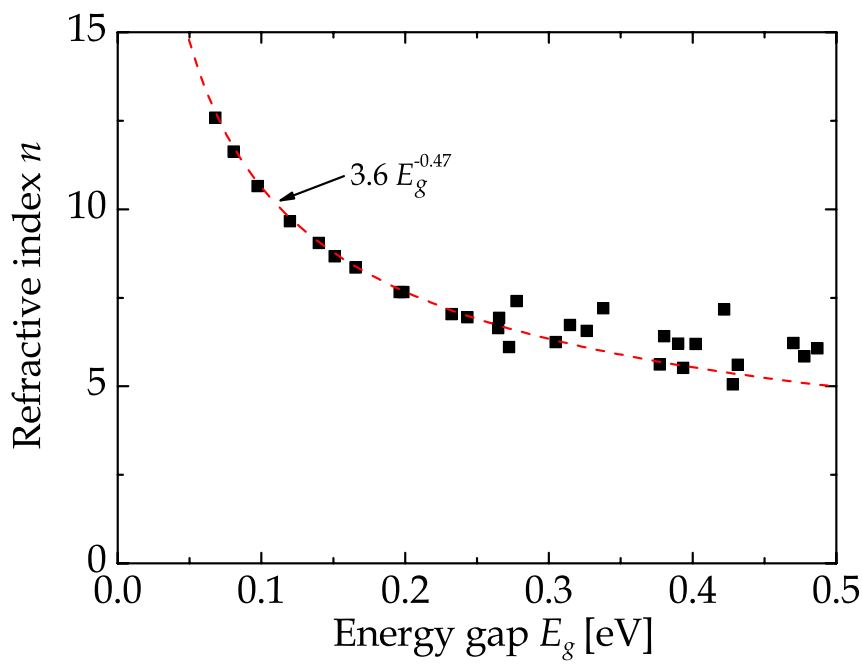

FIG. 8. (Color online) Refractive index at low frequencies vs energy gap for different antidot lattices. The dashed line is a powerlaw fit to the data.

into wave guiding structures fabricated on, e.g., the $\mathrm{SiO}_{2}$ substrate. For all optical and electro-optic applications, the refractive index $n$ is of importance and we wish to study the effect of antidot geometry on $n$. To this end, we compute the complex refractive index via the relation $\widetilde{n}(\omega)$ $=\sqrt{1+i \widetilde{\sigma}(\omega) /\left(d_{g} \varepsilon_{0} \omega\right)}$ for a compilation of different antidot structures with $L$ in the range from 4 to 12 including both large and small energy-gap structures. Below the gap $E_{g}$, the real part of the refractive index dominates and we focus on the real-valued low-frequency $n=\widetilde{n}(0)$ limit. In semiconductors, the refractive index generally decreases with increasing energy gap because more remote electronic transitions make little contribution at low frequencies. A similar tendency is observed in graphene antidot samples, as illustrated in Fig. 8. In the range of small energy gaps, $n$ scales approximately as a power law $E_{g}^{-0.47}$. Hence, tunability of the optical properties also includes the refractive index. The attainable values become very large for low-energy-gap structures approaching the behavior of unmodified graphene.

\section{SUMMARY}

In summary, the optical response of graphene antidot lattices has been analyzed with a $\pi$-electron tight-binding model. We find that these structures behave as dipoleallowed direct-gap two-dimensional semiconductors. The optical properties have been computed using an improved triangle method capable of handling large structures with great accuracy. In addition, inhomogeneous broadening caused by disorder is taken into account. We find that optical infrared spectroscopy is ideally suited for probing the electronic structure. Placing the antidot sample on a dielectric substrate, the response can be probed in both reflection and transmission geometries. We predict clearly visible band-gap features in both modes. Finally, the low-frequency refractive index has been studied for a range of different antidot geometries and we find that the refractive index follows a decreasing power-law behavior with increasing energy gap. 
Note added in proof. Recently, a relevant paper by Nair et $a l .{ }^{25}$ has been published. These authors find additional support for an optical conductivity of $e^{2} / 4 \hbar$ in monolayer graphene. Moreover, for multilayer graphene the transmittance decreases in direct proportion to the number of carbon layers.

\section{APPENDIX: IMPROVED TRIANGLE METHOD}

The triangle method ${ }^{24}$ of approximating two-dimensional integrals of resonant functions is similar to the well-known three-dimensional (3D) tetrahedron method. The key to the method is a linearization of $k$-dependent energies inside small triangular sections of the Brillouin zone. We demonstrate in this appendix that it is possible to include $k$-dependent matrix elements and, thereby, increase the accuracy of response function calculations. We consider an integral of the form

$$
\begin{aligned}
S(\omega) & =\int F(\vec{k}) \delta\left[E_{c v}(\vec{k})-\hbar \omega\right] d^{2} k \\
& =\sum_{\Delta} \int_{\Delta} F(\vec{k}) \delta\left[E_{c v}(\vec{k})-\hbar \omega\right] d^{2} k .
\end{aligned}
$$

Here, " $\Delta$ " denotes a triangle and the sum is over a triangular mesh covering the (irreducible) Brillouin zone. The integral can be reduced to a line integral along the curve $l(\Delta)$ on which $E_{c v}(\vec{k})=\hbar \omega$ according to

$$
S(\omega)=\sum_{\Delta} \int_{l(\Delta)} \frac{F(\vec{k})}{\left|\nabla_{k} E_{c v}(\vec{k})\right|} d l \approx \sum_{\Delta} \frac{1}{\left|\nabla_{k} E_{c v}\right|} \int_{l(\Delta)} F(\vec{k}) d l,
$$

where the linear approximation for $E_{c v}(\vec{k})$ has been assumed. This approximation means further that $l(\Delta)$ becomes a straight line, as illustrated in Fig. 9.

We now invoke the linear approximation for $F(\vec{k})$ as well. Hence, the remaining task is reduced to integrating a linearly varying function along a straight line. We introduce the compact notation $E_{i} \equiv E_{c v}\left(\vec{k}_{i}\right)$ and take the transition energies in the three corners to be ordered according to $E_{0} \leq E_{1} \leq E_{2}$.

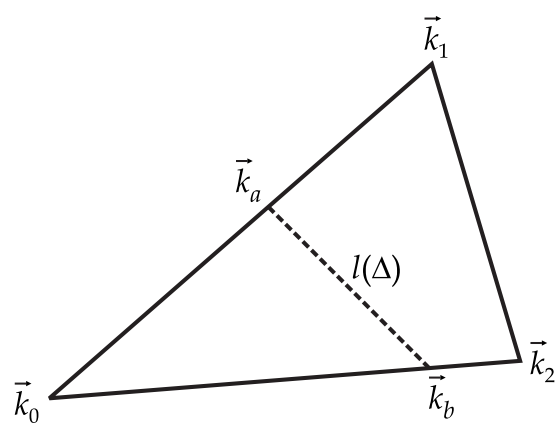

FIG. 9. Important $k$ points used in the improved triangle method.

Provided $E_{0} \leq \hbar \omega<E_{1}$, start $\left(\vec{k}_{a}\right)$ and end $\left(\vec{k}_{b}\right)$ points of the line $l(\Delta)$ are located at (cf. Fig. 9)

$$
\vec{k}_{a}=\vec{k}_{0}+\left(\vec{k}_{1}-\vec{k}_{0}\right) \frac{\hbar \omega-E_{0}}{E_{10}}, \quad \vec{k}_{b}=\vec{k}_{0}+\left(\vec{k}_{2}-\vec{k}_{0}\right) \frac{\hbar \omega-E_{0}}{E_{20}},
$$

with $E_{i j} \equiv E_{i}-E_{j}$. A slightly different expression for $\vec{k}_{a}$ is found for the case $E_{1} \leq \hbar \omega<E_{2}$. Hence, the integral in Eq. (A2) becomes

$$
S(\omega) \approx \sum_{\Delta} \frac{l(\Delta)}{2\left|\nabla_{k} E_{c v}\right|}\left[F\left(\vec{k}_{a}\right)+F\left(\vec{k}_{b}\right)\right] .
$$

Using simple algebra, all quantities may be expressed in terms of values in the three corners and the triangle area $A_{\Delta}$ and we can finally write $S(\omega) \approx \Sigma_{\Delta} S_{\Delta}(\omega)$ with

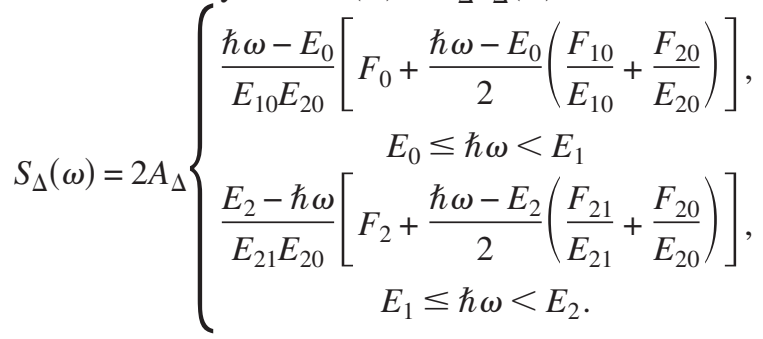

This expression allows us to evaluate resonant integrals using relatively few $k$ points and retaining great accuracy.
${ }^{1}$ K. S. Novoselov, A. K. Geim, S. V. Morozov, D. Jiang, Y. Zhang, S. V. Dubonos, I. V. Grigorieva, and A. A. Firsov, Science 306, 666 (2004).

${ }^{2}$ A. K. Geim and K. S. Novoselov, Nat. Mater. 6, 183 (2007).

${ }^{3}$ L. A. Ponomarenko, F. Schedin, M. I. Katsnelson, R. Yang, E. W. Hill, K. S. Novoselov, and A. K. Geim, Science 320, 356 (2008).

${ }^{4}$ M. Y. Han, B. Özyilmaz, Y. Zhang, and P. Kim, Phys. Rev. Lett. 98, 206805 (2007).

${ }^{5}$ Y. Son, M. L. Cohen, and S. G. Louie, Nature (London) 444, 347 (2006)

${ }^{6}$ E. W. Hill, A. K. Geim, K. Novoselov, F. Schedin, and P. Blake, IEEE Trans. Magn. 42, 2694 (2006).
${ }^{7}$ C. Berger, Z. Song, X. Li, X. Wu, N. Brown, C. Naud, D. Mayou, T. Li, J. Hass, A. N. Marchenkov, E. H. Conrad, P. N. First, and W. A. de Heer, Science 312, 1191 (2006).

${ }^{8}$ S. Russo, J. B. Oostinga, D. Wehenkel, H. B. Heersche, S. S. Sobhani, L. M. K. Vandersypen, and A. F. Morpurgo, Phys. Rev. B 77, 085413 (2008).

${ }^{9}$ T. G. Pedersen, C. Flindt, J. Pedersen, N. A. Mortensen, A. P. Jauho, and K. Pedersen, Phys. Rev. Lett. 100, 136804 (2008).

${ }^{10}$ D. R. Hofstadter, Phys. Rev. B 14, 2239 (1976).

${ }^{11}$ T. G. Pedersen, K. Pedersen, and T. B. Kriestensen, Phys. Rev. B 63, 201101(R) (2001).

${ }^{12}$ T. G. Pedersen, Phys. Rev. B 67, 113106 (2003).

${ }^{13}$ R. Saito, G. Dresselhaus, and M. S. Dresselhaus, Physical Prop- 
erties of Carbon Nanotubes (Imperial College, London, 1998).

${ }^{14}$ J. Cserti, Phys. Rev. B 75, 033405 (2007).

${ }^{15}$ L. A. Falkovsky and A. A. Varlamov, Eur. Phys. J. B 56, 281 (2007).

${ }^{16}$ L. A. Falkovsky and S. S. Pershoguba, Phys. Rev. B 76, 153410 (2007).

${ }^{17}$ H. Haug and S. W. Koch, Quantum Theory of the Optical and Electronic Properties of Semiconductors (World Scientific, Singapore, 1993).

${ }^{18}$ P. Blake et al., Appl. Phys. Lett. 91, 063124 (2007).

${ }^{19}$ S. Roddaro, P. Pingue, V. Piazza, V. Pellegrini, and F. Beltram, Nano Lett. 7, 2707 (2007).

${ }^{20}$ D. S. L. Abergel, A. Russell, and V. I. Falko, Appl. Phys. Lett.
91, 063125 (2007).

${ }^{21}$ J. Matsuoka, N. Kitamura, S. Fujinaga, T. Kitaoka, and H. Yamashita, J. Non-Cryst. Solids 135, 86 (1991).

${ }^{22}$ Handbook of Optical Constants of Solids, edited by E. D. Palik (Academic, New York, 1985).

${ }^{23}$ F. Wang, Y. Zhang, C. Tian, C. Girit, A. Zettl, M. Crommie, and Y. R. Shen, Science 320, 206 (2008).

${ }^{24}$ O. Pulci, B. Adolph, U. Grossner, and F. Bechstedt, Phys. Rev. B 58, 4721 (1998).

${ }^{25}$ R. R. Nair, P. Blake, A. N. Grigorenko, K. S. Novoselov, T. J. Booth, T. Stauber, N. M. R. Peres, and A. K. Geim, Science 320, 1308 (2008). 